\title{
Metodología 5S como herramienta para mejorar la productividad en las empresas
}

5S methodology as a tool to improve productivity in companies

1 Betsy Mabel Moran Olvera

Universidad de Guayaquil, Guayaquil, Ecuador

https://orcid.org/0000-0003-4644-8209

betsy.olveram@ug.edu.ec

2 Yelena Tamara Chávez Cujilán

Universidad de Guayaquil, Guayaquil, Ecuador

https://orcid.org/0000-0002-3989-9936

yelena.chavezc@ug.edu.ec

Artículo de Investigación Científica y Tecnológica

Enviado: 16/12/2021

Revisado: 25/12/2021

Aceptado: 28/01/2022

Publicado:05/02/2022

DOI: https://doi.org/10.33262/ap.v4i1.1.164

Cítese: Moran Olvera, B. M., \& Chávez Cujilán, Y. T. (2022). Metodología 5S como herramienta para mejorar la productividad en las empresas . AlfaPublicaciones, 4(1.1), 358-371. https://doi.org/10.33262/ap.v4i1.1.164

ALFA PUBLICACIONES, es una Revista Multidisciplinar, Trimestral, que se publicará en soporte electrónico tiene como misión contribuir a la formación de profesionales competentes con visión humanística y crítica que sean capaces de exponer sus resultados investigativos y científicos en la misma medida que se promueva mediante su intervención cambios positivos en la sociedad. https://alfapublicaciones.com

La revista es editada por la Editorial Ciencia Digital (Editorial de prestigio registrada en la Cámara Ecuatoriana de Libro con No de Afiliación 663) www.celibro.org.ec 
Palabras

claves: $5 \mathrm{~S}$,

mejora

continua, productividad.

\section{Keywords:}

$5 \mathrm{~S}$, continuous improvement, productivity.

\section{Resumen}

En búsqueda de la excelencia en todas las operaciones y transacciones comerciales, las empresas hoy en día indagan en procesos más seguros, con menos pérdidas y que generen más ganancias, por lo que se hace necesario reorganizarse y aplicar estrategias como el Kaizen y sus herramientas, entre ellas, la 5S. La metodología $5 \mathrm{~S}$ proviene de los términos en japonés de los cinco elementos básicos del sistema: Seiri (selección), Seiton (sistematización), Seiso (limpieza), Seiketsu (normalización) y Shitsuke (autodisciplina). El objetivo de este documento es exponer el marco conceptual de las $5 \mathrm{~S}$ y estudiar las etapas de la implementación de esta metodología, para la mejora continua de la calidad y productividad en las empresas específicamente en los puestos de trabajo. El tipo de investigación realizada fue documental, lo que permitió estudiar los puntos de vistas de diferentes autores en el contexto internacional para evidenciar la importancia de la aplicación de las $5 \mathrm{~S}$ en las organizaciones, además de revisar los principales beneficios de su implementación. Se concluye que poniendo en práctica la metodología $5 \mathrm{~S}$ se logra el compromiso del mejoramiento continuo de la calidad, la productividad, así como también se increméntala competitividad, al ofrecer productos y servicios de calidad.

$5 \mathrm{~S}$, mejora continua, productividad.

\section{Abstract}

In search of excellence in all operations and commercial transactions, companies today are investigating safer processes, with fewer losses and that generate more profits, so it is necessary to reorganize and apply strategies such as Kaizen and its tools, among them, the $5 \mathrm{~S}$. The $5 \mathrm{~S}$ methodology comes from the Japanese terms of the five basic elements of the system: Seiri (selection), Seiton (systematization), Seiso (cleanliness), Seiketsu (normalization) and Shitsuke (self-discipline). The objective of this document is to expose the conceptual framework of the $5 \mathrm{~S}$ and study the stages of the implementation of this methodology, for the improvement continuous quality and productivity in companies specifically in jobs. The type of research carried out was documentary, which made it possible to study the points of view of different authors in the international context to demonstrate the importance of the application of the $5 \mathrm{~S}$ in organizations, in addition 
to reviewing the main benefits of its implementation. It is concluded that by putting the $5 \mathrm{~S}$ methodology into practice, the commitment to continuous improvement of quality and productivity is achieved, as well as increasing competitiveness, by offering quality products and services.

\section{Introducción}

Actualmente es indispensable para las empresas que desean subsistir, que éstas sean competitivas; para ello se requiere experimentar un mejoramiento continuo de sus prácticas, y contar con la colaboración de todas las personas que en ellas laboran. Para ello las empresas incorporan en su planificación estratégica y operativa, objetivos relacionados al mejoramiento continuo de la calidad y la productividad, con el propósito de alcanzar mejores niveles de competitividad en el mercado.

Es así como la cultura de la mejora continua ha tenido impacto en la dinámica empresarial, permitiendo que las organizaciones establezcan parámetros para medir la calidad tanto en los productos y servicios que otorgan. Las empresas al carecer de la cultura de mejora continua, se evidencia en el comportamiento y acciones que realizan los colaboradores. Lo anterior se hace notable en el área de producción, manifestándose mediante el desorden y la falta de aseo, lo que genera diversas consecuencias.

Por ejemplo, Japón es referencia de caso de estudio, ya que, desde el inicio de la década de los años 80, por el surgimiento de un nuevo modelo de administrar las empresas direccionaron a una cultura de la calidad donde se dio el compromiso en todos los niveles gerenciales y operativos con la satisfacción total de sus clientes. Luego de la 2 da guerra mundial, se dio la evolución de la cultura de calidad japonesa y de sus productos a nivel mundial, y de la influencia del pensamiento de W. Edwards Deming

desde 1951, quien indujo y mostro a los japoneses que es necesario la constancia de propósito y el liderazgo para el trabajo en equipo en todos los niveles de la organización, para el logro de la satisfacción del cliente (Deming, 1989).

Es de mencionar, que según Piñero et al. (2018) el éxito de la gestión empresarial en las organizaciones japonesas se debe a las características de sus directivos, gerentes y trabajadores, tales como: constancia, dedicación, organización y

disciplina, lo que conlleva al logro de sus metas y así obtener mejora continua de la calidad, la productividad y la competitividad. La aplicación de las técnicas japonesas constituye una excelente oportunidad de benchmarking de buenas prácticas en gestión, para la mejora de la calidad y la productividad en las industrias latinoamericanas. 
Ahora bien, las empresas japonesas aplicaron la filosofía de gestión de calidad conocida con el nombre Toyota Production System (TPS), en su traducción al español, Sistema de Producción Toyota, para las décadas de los 50, 60 y 70 lideradas por Taiichi Ohno, quien fue un alto ejecutivo del grupo Toyota. Son diferentes las técnicas empleadas en la filosofía de gestión del TPS para la gerencia de la mejora continua (Kaizen) de la calidad y la productividad en el sitio o puestos de trabajo (Gemba) (Piñero et al., 2018).

Hay varias técnicas del TPS Omogbai \& Salonitis (2017) entre las cuales se encuentran: 5S, PokaYoke, Just InTime, Kanban, Jidoka, Muda, Mieruka, Mantenimiento Autónomo, Círculos de Controles de Calidad, Herramientas Básicas y Administrativas de la Calidad y Metodología del Ciclo Planificar, Hacer, Verificar y Acción (PHVA). Estas técnicas son aplicadas no solamente en el sector automotriz sino también en los diferentes sectores industriales, incluyendo las organizaciones del sector servicio.

En atención al contexto anterior, el objetivo de este documento es exponer el concepto de las $5 \mathrm{~S}$ y estudiar las etapas de la implementación de esta metodología, para la mejora continua de la calidad y productividad en las empresas específicamente en los puestos de trabajo, así como revisar los principales beneficios de su implementación.

De esta manera, 5S denota cinco operaciones básicas y elementales que toda compañía camino a la excelencia debe seguir: Seiri - Seiton, Seiso - Seiketsu - Shitsuke que en el idioma español se traduce como selección - Orden - Limpieza - Estandarización y Disciplina respectivamente; y por sus iniciales japonesas dan origen al nombre de esta metodología.

La metodología de las 5S es parte de las técnicas del Sistema de Gestión de la Producción, cada una de las técnicas se interrelacionan en el proceso de la mejora continua en las empresas. El logro de los resultados depende del liderazgo de la alta gerencia, y de la participación y compromiso de todo el equipo humano de organización.

\section{Metodología}

La metodología que se usó en la investigación para este artículo es la documental, ya que permitió al investigador familiarizarse con la temática que se estudia y revisar conceptos de la metodología $5 \mathrm{~S}$ y mejora continua.

De lo anteriormente expuesto, se cita a Hernández \& Fernández (2014), quienes definen la investigación documental como: “detectar, obtener y consultar la bibliografía y otros materiales que parten de otros conocimientos y/o informaciones recogidas moderadamente de cualquier realidad, de manera selectiva, de modo que puedan ser útiles para los propósitos del estudio". 
De forma similar, Losada \& López (2003) conceptualizan la investigación bibliográfica como una técnica cualitativa que se encarga de explorar todo aquello que se haya escrito acerca un determinado tema o problema. Este tipo de investigación pretende cumplir como basamento teórico del trabajo que se quiere realizar, así como evitar que las investigaciones sean réplicas de alguna ya hechas.

La investigación documental se debe enfocar exclusivamente en el tema de estudio sin perderse en otros temas ajenos al mismo. Para este artículo, se recopiló información de tipo bibliográfico, es decir, documentos escritos como: libros, artículos de publicaciones como revistas científicas y artículos que se obtuvieron de portales de internet con especialidad en el tema.

A partir de la obtención de la información, esta fue analizada objetivamente por el investigador, la cual permitió exponer el marco conceptual de las 5S, estudiar las etapas de la implementación de esta metodología y además de revisar los principales beneficios de su implementación en las empresas para la mejora continua de la calidad y productividad.

\section{Resultados y Discusión}

\section{Metodología de las 5S}

La filosofía Kaizen, aplicada por la Toyota, es el caso de éxito mundial más emblemático y de muestra que con esta metodología es posible elevar los estándares de productividad en las empresas (Salazar et al., 2020). En Latinoamérica aún estas técnicas son poco conocidas. Bajo este concepto, el tiempo ha demostrado que los japoneses no se equivocaron, ya que Japón es un país altamente industrializado, tiene las mejores economías del planeta y es altamente competitivo.

Cuando se hace mención al mejoramiento continuo, de inmediato se habla de Kaizen, que significa mejoramiento continuo y estos mejoramientos van de pequeños e incrementales a innovaciones drásticas y radicales, involucrando a todas las personas tanto gerentes como trabajadores lo que origina resultados favorables a través del tiempo (Imai, 2006). Para este autor, la filosofía kaizen se convirtió en la clave de la ventaja competitiva japonesa, mientras que as $5 \mathrm{~S}$ tienen como fin lograr un mayor orden, eficiencia, y disciplina en el lugar de trabajo

La metodología 5S, es un acrónimo para ordenar, establecer en orden, brillo, estandarizar y sostener, es una de esas herramientas, la cual se les da nombres diferentes en la literatura, pero significan lo mismo Omogbai \& Salonitis (2017). En otras palabras, se refiere a una estrategia cuyo objetivo es minimizar la cantidad de tiempo y recursos utilizados en los procesos de fabricación y otras actividades de una empresa, haciendo énfasis en eliminar todas las formas de desperdicio. 
Las $5 \mathrm{~S}$ es una herramienta mundialmente conocida implantada inicialmente en las industrias japonesas. Esta metodología impactó y generó cambios tanto en las empresas como en las personas que la aplican, porque se centran en potenciar el aprendizaje de las personas que trabajan en las organizaciones gracias a su simplicidad y agilidad por realizar pequeños cambios y mejoras con el fin de experimentar y aprender con ellas (Aldavert et al., 2016).

Las 5S pueden representarse como un sistema que posibilita la creación de las condiciones necesarias para la implementación de nuevas soluciones técnicas; se basa en ideas innovadoras, la optimización del espacio de trabajo y el proceso de producción se realizan también; adopta un enfoque sistemático que implica el trabajo en equipo, incluyendo la participación de todos los empleados, y se centra en la aplicación total de la organización del trabajo y la adaptación del espacio de trabajo (Vorkapić et al., 2017).

En la aplicación de la metodología de las 5S se debe contar con el alto involucramiento de la gerencia general de la empresa, además de capacitación para todos sus miembros desde el gerente hasta el colaborador del último nivel jerárquico. Carvalho de Souza et al. (2018) manifiestan que la implementación y mantenimiento del programa 5S no es una tarea sencilla, debido a que está directamente relacionado con el comportamiento y la cultura de las personas, hecho por el cual muchas empresas no logran los resultados esperados al implementar esta estrategia.

La metodología 5S es una herramienta de mejora en las empresas, enfocada a buenas prácticas y tiene que ver con el Ciclo Deming (Ciclo de mejora continua PHVA) y mejorar la efectividad de los procesos operativos.

Las 5S provienen de 5 palabras japonesas: SEIRI: Seleccionar, SEITON: Ordenar, SEISO: Limpiar, SEIKETSU: Estandarizar, SHITSUKE: Disciplina. Seguidamente de describe cada $\mathrm{S}$ en atención a lo propuesto por (Contreras \& Zare, 2019).

\section{SEIRI (Clasificar, Seleccionar)}

Se refiere a que en todas las áreas del trabajo se debe separar elementos innecesarios de los que son necesarios y posteriormente descartar lo innecesario. $5 \mathrm{~S}$ el primer paso es inspeccionar las zonas de trabajo y eliminar los materiales que innecesarios para la realización del trabajo. Del mismo modo, se deben analizar las actividades y eliminar las tareas que no producen resultados.

El propósito de la selección es retirar del ambiente de trabajo todos los elementos innecesarios para las operaciones cotidianas, manteniendo así cerca los elementos necesarios que facilitan la labor del trabajo ya que de esta manera se eliminan obstáculos que pueden con-llevar a paradas innecesarias de las actividades del trabajo. 


\section{SEITON (Ordenar)}

Colocar lo necesario en sitios accesibles, según la frecuencia y secuencia de uso, es decir, consiste en establecer el modo en que deben ubicarse e identificarse los materiales necesarios, de manera que sea fácil y rápido encontrarlos, utilizarlos y reponerlos.

Una vez eliminados los materiales (y tareas) innecesarios, hay que ordenar lo que realmente se necesite para trabajar. Cada herramienta, materia prima, debe tener un lugar asignado y único. Haciendo esto se consigue evitar la pérdida o extravío de material y ahorra tiempo muerto buscando cosas que utilizamos frecuentemente (Contreras \& Zare, 2019).

Ordenar tiene como propósito reducir los tiempos de búsqueda, reducir movimientos innecesarios dentro del área de labores, evitar confusiones peligrosas, permitir la ubicación de materiales, documentos de forma rápida; un ambiente ordenado da la impresión de que todo marcha bien, que las cosas se hacen bien y por lo tanto el producto final es de buena calidad; mejora el manejo de existencias y la coordinación para la ejecución de las tareas (Salazar et al., 2020).

\section{SEISO(Limpiar)}

Consiste en limpiar e inspeccionar el sitio de trabajo y los equipos para prevenir la suciedad.

Implementando acciones para evitar o disminuir la suciedad y hacer más seguro los ambientes de trabajo no solo es ir a quitar polvo y limpiar, también implica determinar las causas por las cuales no se mantiene limpia el área, de tal manera que se pueda solucionar el problema de raíz, evitando que se repita la misma situación (Zubia et al., 2018).

Para ello se recomienda fijar tareas periódicas que consigan que las zonas queden despejadas y solamente con los materiales necesarios, sin ningún otro tipo de material que pueda entorpecer las actividades (Contreras \& Zare, 2019).

\section{SEIKETSU (Estandarizar)}

Es el mantenimiento de las condiciones conseguidas. Por lo tanto, se debe mantener los uniformes limpios, aseo personal correcto y de las áreas de trabajo. Se sugiere el desarrollo de normas de limpieza las cuales sean claras y específicas, también se puede hacer un procedimiento en el cual sea visible para todos y explique de manera clara cuáles son los lineamientos para mantener el área en orden y limpia (Zubia et al., 2018).

\section{SHITSUKE (Disciplina o mejora continua)}


Para complementar las 5S se debe asumir la filosofía de mejora continua, basada en el conocido ciclo PHCA (Planificar -> Hacer -> Controlar -> Actuar) que indica "dado que nada es perfecto, siempre queda margen para mejorar". Se deben realizar periódicamente evaluaciones del estado actual de cada proceso clave para encontrar posibles deficiencias y proponer mejoras en las actividades para conseguir mejores resultados (Contreras \& Zare, 2019). Significa que se debe de evitar a toda costa que no se4 sigan los procedimientos ya establecidos en la organización con el fin de que la limpieza sea parte de la cultura de la empresa (Zubia et al., 2018).

De lo anterior, se puede inferir que la metodología de las $5 \mathrm{~S}$ es una herramienta sencilla, aplicable y con resultados evidentes a bajo costo, genera una buena imagen hacia los clientes, así como un sano ambiente de trabajo, promueve la productividad al contar con materiales, mobiliarios y equipos necesarios, ordenados y limpios, además genera en los trabajadores la adopción de la cultura de mejora continua.

\section{Etapas de la implementación de la Metodología $5 S$}

La implementación de las 5S se da en una etapa para cada una de las "S", ya que cada una implica una mejora en el proceso. Para ello cada grupo de trabajo determina las necesidades y tareas que deben realizar cada una de las "S".

Según Salazar et al. (2020), el primer paso para la implementación es la reunión de inicio, la misma busca informar sus objetivos, capacitar a los colaboradores, diagnosticar la situación inicial y establecer las oportunidades de mejora. Este paso debe ser liderizado por un responsable de la implementación quien debe ser autorizado por un representante de la alta dirección.

El segundo paso es el proceso de implementación que, como tal, establece las oportunidades de mejora en el primer paso, se organizan los equipos de trabajo que se encargarán de realizar las acciones para la mejora del proceso. En este caso, el líder, da las directrices y hace el seguimiento a las diferentes actividades programadas en el primer paso. En este paso se debe hacer uso de herramientas según las actividades y el registro fotográfico del antes, es fundamental para tener una orientación de cómo se está para después comparar con la mejora propuesta y establecer indicadores de gestión.

La etapa tres es de evaluación y control. Con el establecimiento de indicadores se hace la evaluación, por lo que es fundamental medir el nivel de implementación de las 5S. Se puede hacer uso de hojas de cálculo que son fáciles de manejar para llevar un control. La evaluación periódica utilizando instrumentos y formatos de registro sencillos, establecidos según la necesidad, sirven para evaluar el nivel de implementación y tiene como base la información levantada durante las inspecciones de rutina para las $5 \mathrm{~S}$ (Salazar et al., 2020). 
El cuarto paso de la implementación es la difusión. Este paso es muy importante ya que, al ser un proceso de mejora, se debe difundir los resultados para mostrar el trabajo de los colaboradores y motivar a los mismos a continuar con el proceso. Se sugiere hacer uso por ejemplo de murales, buzón de sugerencias que son alternativas muy buenas para llevar a cabo esta etapa.

De manera similar para implementar la Metodología 5S, Zubia et al. (2018) proponen que se deben seguir una serie de pasos dispuestas en la tabla 1

\section{Tabla 1}

\section{Pasos para implementar la metodología 5S}

Etapa

1. Diagnostico

2. Concientizar

3. Capacitación

4. Plan de Implementación de las $5 \mathrm{~S}$

5. Implementación del 5S

Descripción

El objetivo de esta etapa es elaborar un diagnóstico de la empresa, mediante entrevistas a los colaboradores y observación directa de las actividades que se desarrollan en ella.

Consiste en concientizar al personal respecto a la importancia de la metodología de las 5S, mediante la sensibilización del tema, buscando generar en los colaboradores una necesidad por la metodología.

Se realiza mediante un taller práctico sobre la implementación de la metodología. Se debe entregar un manual a cada colaborador, en el cual se incluyeron los temas de los objetivos y beneficios.

Se debe realizar una planeación previa a la implementación, en la cual se les proporcionó a los colaboradores sus responsabilidades a ejecutar.

se aplica las 5S de la metodología: 1) organización (seiri), 2) orden (seiton) 3) limpieza (seisou), 4) estandarización (seiketsu) y 5) disciplina (shitsuke).

Fuente: Elaboración propia basado en (Zubia et al., 2018)

El método de las 5S trata de lograr lugares de trabajo mejor organizados, más ordenados y más limpios de forma permanente para conseguir una mayor productividad y entorno laboral seguro.

Al implementar las 5S se crean condiciones necesarias para la generación de nuevas soluciones técnicas e innovadoras, donde se optimiza el espacio de trabajo y el proceso de producción adopta un enfoque sistemático que implica el trabajo en

equipo, incluyendo la participación de todos los empleados, y se centra en la aplicación total de la organización y la adaptación del espacio de trabajo (Vorkapić et al., 2017). 
Es de tal relevancia la metodología de las 5S, que en Latinoamérica existen en algunos países con la experiencia de los Premios Nacional 5S es el caso de Argentina, Perú y México, estos premios 5S cuentan con el respaldo de The Association for Overseas Technical Cooperation and Sustainable Partnerships (AOTS), Japan. (AOTS, 2016).

En el contexto internacional y en Latinoamérica, se puede evidenciar el interés en el tema de las 5S, y en su implantación, como primer paso en la búsqueda de la excelencia empresarial. En el caso de Ecuador, representa una

prioridad su promoción y recomendaciones de iniciar y consolidar las experiencias de las 5S para lograr el compromiso del mejoramiento continuo de la calidad, la productividad en los puestos de trabajo, con un mejor ambiente laboral con seguridad y salud labora (Piñero et al., 2018).

La metodología 5S fue ampliada a 6S por la adición de "seguridad" Dhounchak \& Khatak (2017), refiriéndose a la seguridad en el lugar de trabajo, en cuyo proceso, además de simplificar el ambiente de trabajo, reducir las actividades de desecho y sin valor, al tiempo que, además de mejorar la eficiencia de la calidad, se incluye también la seguridad.

En este orden de ideas, en las 6S, la seguridad es una manera de mantener al trabajador y al lugar de trabajo seguros: Se centra en la eliminación de los peligros y la creación de un entorno de trabajo seguro, bajo la premisa de que es fácil reconocer peligros potenciales cuando el lugar de trabajo está bien organizado y limpio, y que se debe realizar un "barrido de seguridad" separado para identificar, etiquetar y hacer frente a los peligros (Reyes et al., 2017).

También, las 5S como cualquier otra metodología está diseñada para optimizar la productividad, el vínculo de estos dos aspectos está respaldado por actividades trasversales para su correcta funcionalidad, entre las cuales se puede destacar el liderazgo de la parte directiva de una empresa, la claridad de los objetivos y el criterio de evaluación. La metodología permite la disminución de defectos en la cadena de producción (Yantalema, 2020).

\section{Beneficios de la estrategia de las $5 S$}

Las 5S, reducen elementos innecesarios de trabajo, facilita el acceso y devolución de objetos u elementos. Evita la pérdida de tiempo en la búsqueda de elementos en lugares no organizados ni apropiados, reducción de fuentes que originan suciedad, mantiene las condiciones necesarias para el cuidado de las herramientas, equipo, maquinaria, mobiliario, instalaciones y otros materiales, entorno visualmente agradable, creación y mantenimiento de condiciones seguras para realizar el trabajo, mejora el control visual de elementos de trabajo, crea las bases para incorporar nuevas metodologías de 
mejoramiento continuo, es aplicable en cualquier tipo de trabajo: manufactura o de servicio y participación en equipo

De igual manera Eurofins Envira Ingenieros Asesores (2020), indican que la mejora continua es una tarea de todos, dado que la implantación del método de "las $5 \mathrm{~S}$ " se basa en el trabajo en equipo involucrando a todos en el proceso de mejora desde su conocimiento del puesto de trabajo. La calidad empieza por los trabajadores y por el ambiente que le rodea. Esta es la razón de la utilización de la metodología $5 \mathrm{~S}$ donde se respire un ambiente eficiente, seguro y confortable y que se aprecie los siguientes beneficios:

- Mayor productividad.

- Genera cultura organizacional y disciplina personal.

- Mejora de manera significativa el entorno físico del puesto de trabajo, aumentando el orden y el espacio

- Mejora las condiciones de trabajo, aumentando los niveles de seguridad y disminuyendo los accidentes.

- Reducción de pérdidas de tiempo y tiempos de respuesta más cortos.

- Mejora la imagen ante los clientes.

- Mejora en la calidad de productos y/o servicios.

- Genera mayor compromiso, responsabilidad, cooperación y trabajo en equipo.

- Acerca a la organización a implementar gestión de calidad total.

Finalmente, la importancia de la metodología 5 s es que al aplicarse puede llegar a marcar diferencia con otras organizaciones en cuanto a la reducción de tiempos y por ende enfrentarlos cambios que en la actualidad exigen las instituciones en el mundo globalizado pues no solamente se debe de estar preparado hacia el interior sino también hacia los factores externos que influyen, ya sea de manera directa o indirecta, en las organizaciones y que a la vez son un reto para la competitividad en el mundo (Pérez, 2021).

\section{Conclusiones}

- La aplicación de la metodología de las 5S en las empresas permite mejorar la cultura de calidad en el equipo productivo, así como se incrementa de manera significativa el orden y limpieza en el área de trabajo.

- Se describió el marco conceptual de las 5S y los resultados ilustran que al implementarla en las empresas se obtiene ahorro en los costos de operación, una eficaz gestión de los recursos, reducción de accidentes, mejora en el clima laboral debido al aumento de motivación del personal al encontrarse más a gusto en su lugar de trabajo. Hay reducción de desperdicios y tiempos de esperas, se optimiza los espacios y se alarga la vida útil de equipos y herramientas de trabajo 
- Las etapas para implantar las 5S son el diagnostico, la concientización, capacitación, plan de implementación y la implementación como tal.

- La medida de productividad se incrementa al aplicar la filosofía de las 5Sreduciendo los tiempos omitidos y perdidos durante los procesos; generando un incremento de las horas efectivas y reduciendo costos de operación

Por último, poniendo en práctica la metodología $5 \mathrm{~S}$ se logra el compromiso del mejoramiento continuo de la calidad, la productividad, así como también se increméntala competitividad, al ofrecer productos y servicios de calidad.

\section{Referencias Bibliográficas}

Aldavert, J., Aldavert, X., Vidal, E., \& Lorente, J. (2016). 5S para la Mejora Continua. Barcelona: Editorial Cims (C) Midac.

AOTS. (2016). Premio Nacional 5s.

Carvalho de Souza, B., Alcantara, G., \& Parreira, P. (2018). Implementación del programa $5 S$ a través de la metodología DMAIC.

Contreras, I., \& Zare, V. (2019). La metodología 5 S como herramienta de mejora en las empresas industriales, de Latinoamérica, en los últimos 5 años: una revisión de literatura científica. Trabajo especial de grado de la Universidad Privada del Norte.

Deming, E. (1989). Calidad, Productividad, Competitividad. La salida de la Crisis. Madrid: Ediciones Diaz Santo.

Dhounchak, D., \& Khatak, N. (2017). 6 S Methodology and its applications. International Journal of Research in Mechanical Engineering , 46-58.

Eurofins Envira Ingenieros Asesores. (25 de 04 de 2020). ¿En qué consiste el método de las $5 S$ ?

Hernández, R., \& Fernández, C. (2014). Metodología de la Investigación. México: McGraw Hill.

Imai, M. (2006). Como Implementar el Kaizen en su sitio de trabajo (Gemba). Bogotá: McGrawHill.

Losada, J., \& López, R. (2003). Métodos de Investigación en ciencias humanas y sociales. Barcelona: Paraninfo- Thomson.

Omogbai, O., y Salonitis, K. (2017). The implementation of 5S lean tool using system dynamics approach. Procedia CIRP, 380 - 385. 
Pérez, C. (2021). Metodología 5S y su impacto en la reducción de tiempos a nivel empresarial.

Piñero, E., Vivas, F., \& Flores, L. (2018). Programa 5S's para el mejoramiento continuo de la calidad y la productividad en los puestos de trabajo. Ingeniería Industrial. Actualidad y Nuevas Tendencias, Universidad de Carabobo. Venezuela, 99 - 110.

Reyes, J., Aguilar, L., Hernández, J., \& Mejias, A. (2017). La Metodología 5S como estrategia para la mejora continua en industrias del Ecuador y su impacto en la Seguridad y Salud Laboral. Polo del Conocimiento, 1040 - 1059.

Salazar, C., Ore, H., Benavides, B., Delgado, J., \& Pantoja, L. (2020). Metodología 5S, alternativa viable en la mejora de procesos de la industria alimentaria. Rev. Tayacaja. 114 - 124.

Vorkapić, M., Cockalo, D., Dordević, D., \& Besić, C. (2017). Implementation of 5 s tools as a starting point in business process reengineering. Journal of Engineering Management and Competitiveness, 44 - 54.

Yantalema, O. (2020). Implementación de la metodología 5 S en el taller mecánico de una industria de alimentos ubicada en Guayaquil.

Zubia, S., Brito, J., \& Ferreiro, V. (2018). Mejora Continua: Implementación de las 5S en una microempresa. Revista Global de Negocios. IBFR 6(5), 97-v110.

\section{【Ciencia}


El artículo que se publica es de exclusiva responsabilidad de los autores y no necesariamente reflejan el pensamiento de la Revista Alfa Publicaciones.

\section{Ciencia}

El artículo queda en propiedad de la revista y, por tanto, su publicación parcial y/o total en otro medio tiene que ser autorizado por el director de la Revista Alfa Publicaciones.
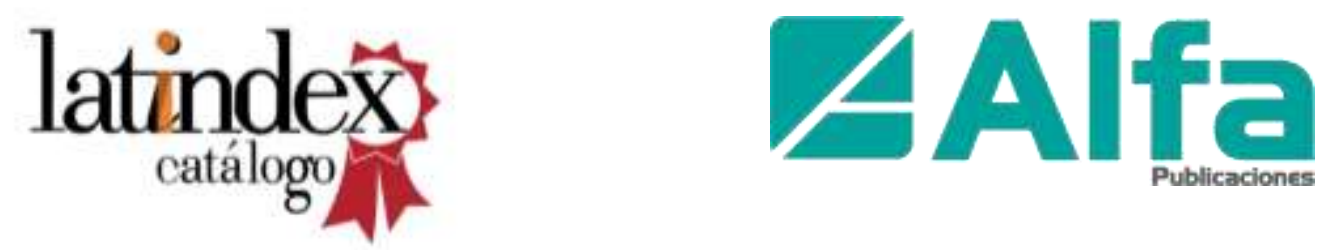

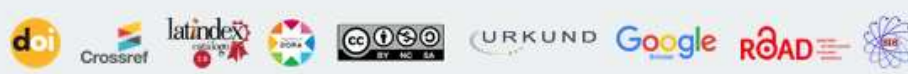
DLatinREV

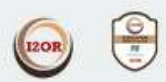

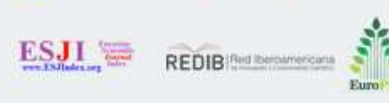

$\underbrace{}_{\text {wizdom.ai }} \mathrm{O}_{\text {OpenAIRE }}^{+}$

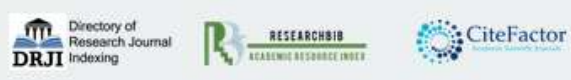

